\title{
Taxa de gestação em cabras Alpinas e Saanen tratadas com hCG no terceiro dia após o estro
}

[Pregnancy rate and progesterone concentration of Alpine and Saanen goats treated with hCG the third day after estrus]

\author{
C.P. Prosperi, C.A.A. Torres, J.D. Guimarães, J.H. Bruschi, P.A.G. Leite, V.V. Maffili \\ Departamento de Medicina Veterinária - UFLA \\ Campus Universitário - Caixa Postal 37 \\ 37200-000 - Lavras, MG
}

\begin{abstract}
RESUMO
Avaliou-se o efeito da aplicação da gonadotrofina coriônica humana (hCG) sobre a taxa e duração da gestação e sobre a concentração plasmática de progesterona (P4) em cabras lactantes das raças Alpina (83) e Saanen (60), na estação de acasalamento induzida pelo programa de fotoperíodo artificial. Os animais foram distribuídos em dois tratamentos após a cobrição; as cabras do tratamento 1 (T1 controle) receberam solução salina via intramuscular $(1 \mathrm{ml})$, e as do tratamento 2 (T2) receberam 250UI do hCG, no terceiro dia no pós-estro. As taxas de gestação, detectadas por exame ultra-sonográfico, realizado no $35^{\circ}$ após a cobrição, para as cabras das raças Alpina e Saanen controle e tratadas, foram de 80,4; 70,3; 72,7; $81,5 \%$, respectivamente, não diferindo entre os tratamentos e entre as raças $(\mathrm{P}>0,05)$. A taxa média de gestação foi 77,2 e 75,0\% para os animais controle e tratados, respectivamente. A duração da gestação não foi influenciada pelos tratamentos $(\mathrm{P}>0,05)$. As amostras de sangue para análise da concentração plasmática de progesterona $(\mathrm{P} 4)$ foram coletadas de cinco cabras Alpina por tratamento, no dia do estro (dia 0) e aos 3, 8, 15, 21, 42 e 60 dias após o estro. Não houve diferença na concentração plasmática média de $\mathrm{P} 4$ entre os tratamentos $(\mathrm{P}>0,05)$, sendo de $5,84 \mathrm{ng} / \mathrm{ml}$ para $\mathrm{T} 1$ e $5,76 \mathrm{ng} / \mathrm{ml}$ para T2. Observouse diferença em relação aos dias de coleta $(\mathrm{P}<0,05)$. $\mathrm{O}$ hCG aplicado no terceiro dia pós-estro não alterou a taxa de gestação e nem a concentração de $\mathrm{P} 4$.
\end{abstract}

Palavras-chave: cabra, gestação, estro, hCG, progesterona

\begin{abstract}
The experimental objective was to evaluate the effect of human chorionic gonadotropin (hCG) administration on pregnancy rate and gestation length and on serum concentration of progesterone (P4) in Alpine (83) and Saanen (60) dairy goats induced by artificial photoperiod to enter the breeding season. Animals were randomly allocated to two treatments following estrus: in treatment 1 (T1) goats received $1 \mathrm{ml}$ saline solution and in treatment 2 (T2) animals received $250 I U$ of $h C G$ in $1 \mathrm{ml}$ saline intramuscularly during the third day after estrus. Pregnancy rates detected by ultrasound technology 35 days after mating did not differ significantly between treatments $(P>0.05)$. They equaled 80.4 and $70.3 \%$ for Alpine goats and 72.7 and 81.5\% for Saanen goats in $T 1$ and T2, respectively. Overall pregnancy rates were $77.2 \%$ and $75.0 \%$ for the controls and treated animals, respectively. Gestation length was not affected by the hCG treatment. Blood samples were collected from 5 Alpine does from each treatment the day of estrus (day 0) and on days 3, 8, 15, 21, 42 and 60 after estrus, for determination of progesterone concentration in plasma. Means were 5.84 and $5.76 \mathrm{ng} / \mathrm{ml}$ for the control and treated does, respectively, and were not affected by treatment $(P>0.05)$, although concentrations did differ among collection days
\end{abstract}

Recebido em 20 de abril de 2004

Aceito em 11 de fevereiro de 2005

E-mail: prosperi@ufla.br 
$(P<0.05)$. The administration of $h C G$ the third day after estrus did not affect pregnancy rate or $P 4$ concentration and had no beneficial effect on reproduction in the does.

Keywords: goat, pregnancy, estrus, $h C G$, progesterone

\section{INTRODUÇ̃̃O}

O índice reprodutivo pode ser maximizado por várias técnicas de manejo, dentre essas o uso de hormônios. A utilização da gonadotrofina coriônica humana (hCG), sintetizada no início da gestação de mulheres, tem a função de transformar o corpo lúteo $(\mathrm{CL})$ cíclico em corpo lúteo gestacional (verum). Sua subunidade $\alpha$ é similar à subunidade do hormônio luteinizante (LH) em humanos, suínos, bovinos e ovinos (Hafez, 1988). O hormônio folículo estimulante (FSH) é liberado pela hipófise de forma pulsátil durante todo o ciclo estral, promovendo várias ondas de crescimento folicular. Os folículos entram em atresia devido à ação negativa da progesterona (P4) produzida pelo CL sobre o eixo hipotalâmico-hipofisário-gonadal, impedindo a produção de $\mathrm{LH}$, em concentrações adequadas, necessárias para o desenvolvimento completo do folículo e, conseqüentemente, para a ocorrência da ovulação. Com a diminuição da concentração plasmática de progesterona, ocorre a liberação no eixo hipotalâmico-hipofisáriogonadal. Nesse momento, o crescimento folicular completa seu desenvolvimento, culminando com a ovulação do(s) folículo(s) dominante(s).

Ginther e Kot (1994) e Medam et al. (2003) verificaram, em cabras, freqüência maior de quatro ondas de crescimento folicular, emergindo nos dias $0,4,8$ e 14 do ciclo estral, e folículos ovulatórios com média de $6 \mathrm{~mm}$ de diâmetro. $\mathrm{O}$ fenômeno de dominância parece ser mais intenso na primeira e na quarta ondas. Com o desenvolvimento folicular no início do metaestro, a aplicação de hormônios, como o hCG, pode levar à ocorrência da ovulação, com a formação de um novo corpo lúteo e/ou apenas a luteinização do folículo.

Saharrea et al. (1998), ao aplicarem hCG 84 horas após o início do estro de cabras superovuladas com gonadotrofina sérica da égua prenhe (PMSG ou eCG), verificaram maior número de corpos lúteos nas cabras tratadas e concluíram que esse efeito pode ter sido causado pela ação direta do hCG sobre as células do CL em formação ou devido à formação de novos corpos lúteos, e, assim, tem-se a elevação da concentração plasmática de P4. Fonseca (2002) demonstrou a eficiência do hCG em elevar a concentração plasmática de progesterona, em cabras das raças Alpina e Saanen, ao utilizar 250UI de hCG no quinto dia após o estro. Waldron et al. (1999) demonstraram que cabras que apresentaram falhas na formação do $\mathrm{CL}$ tiveram ciclos estrais curtos e redução da taxa de gestação. Dessa forma, o hCG pode ser utilizado para evitar a regressão prematura do $\mathrm{CL}$ e promover a formação de novos corpos lúteos e/ou aumentar o número de células grandes do $\mathrm{CL}$, pela conversão das celulas pequenas, tendo em vista que as primeiras são as maiores produtoras de progesterona. Isso pode assegurar melhor taxa de gestação e, conseqüentemente, melhores índices reprodutivos e produtivos em caprinos. Nessa espécie, é fundamental a presença do CL, pois a placenta por si só não é capaz de produzir concentrações adequadas de progesterona para a manutenção da gestação (Gordon, 1997).

Este estudo teve o objetivo de verificar se a aplicação do hCG em cabras no terceiro dia no pós-estro aumenta a taxa de gestação e a concentração plasmática de progesterona.

\section{MATERIAL E MÉTODOS}

Os animais foram alojados em baias coletivas para 16 animais, com acesso a solário. A alimentação foi à base de silagem de milho e ração concentrada conforme as exigências, com a água e sal mineral fornecidos à vontade.

$\mathrm{O}$ experimento foi realizado entre novembro de 2002 e abril de 2003, durante a estação de acasalamento induzida por fotoperíodo artificial. Utilizaram-se 143 cabras em lactação, sendo 83 da raça Alpina e 60 da raça Saanen, distribuídas aleatoriamente em dois tratamentos, após a cobrição. As cabras foram previamente submetidas a fotoperíodo artificial por 60 dias, para induzir a estação reprodutiva no período 
seguinte (de 60 a 70 dias após a retirada da luz artificial). Os animais do tratamento-controle (T1, 46 cabras Alpinas e 33 Saanen) receberam placebo (solução salina $0,9 \%$ ) em volume de $1 \mathrm{ml}$, idêntico ao volume das $250 \mathrm{UI}$ do $\mathrm{hCG}^{1}$, aplicado no terceiro dia no pós-estro (T2, 37 cabras Alpinas e 27 Saanen), por via intramuscular.

A detecção do estro foi realizada com auxílio de um rufião, duas vezes ao dia, às 7 e às 17 horas, por um período mínimo de 20 minutos. Considerou-se em estro a cabra que apresentava interesse pelo macho, movimento lateral da cauda, secreção e edemaciamento da vulva, inquietação, balidos freqüentes e imobilidade à monta. As fêmeas, levadas ao macho de fertilidade conhecida, foram cobertas, no momento da visualização do estro e 24 horas após a primeira cobrição, caso permanecessem em estro.

As amostras de sangue foram coletadas no dia do estro (dia 0), no dia da aplicação do hCG (dia 3) e nos dias 8, 15, 21, 42 e 60 após o estro. A partir do $60^{\circ}$ dia, foram coletadas amostras de sangue de 21 em 21 dias até 120 dias após o estro, realizadas em 15 animais de cada grupo, por meio de punção da veia jugular, em tubos com vácuo e anticoagulante. As amostras foram colocadas em recipiente próprio, sobre gelo, até a centrifugação a $2.000 \mathrm{~g}$ por 15 minutos em centrífuga refrigerada a $5^{\circ} \mathrm{C}$. O plasma foi armazenado em freezer a $-20^{\circ} \mathrm{C}$ até o momento das análises, que nunca excedeu a duas horas. A concentração plasmática de progesterona $(\mathrm{P} 4)$ foi avaliada por radioimunoensaio em fase sólida (kit comercial $^{2}$ ), em cinco animais por tratamento, cujo valor mínimo de P4 mensurável foi de $0,02 \mathrm{ng} / \mathrm{ml}$.

O diagnóstico de gestação foi realizado por ultrasonografia por via transretal, realizada no $35^{\circ}$ dia após a cobrição, por meio de probe de $5 \mathrm{MHz}$ (Aloka SSD $500^{3}$ ). A duração da gestação foi calculada com base no período entre a data de cobertura e a do parto. Verificou-se também o número de animais que pariram em relação aos diagnosticados positivamente ao $35^{\circ}$ dia, para detectar possíveis perdas fetais.

A dispersão de gestações segundo os subuniversos alvo (raças e tratamentos) foi avaliada pelo teste qui-quadrado (Ayres et al., 2000). A concentração plasmática de P4 foi avaliada para a distribuição normal pelo teste Lilliefors e para homogeneidade de variância pelo teste Cochran. Posteriormente, por análise de variância, as médias foram comparadas pelo teste SNK, a 5\%, por meio do pacote computacional SAEG (Ribeiro Júnior, 2001). Buscou-se o ajustamento de uma regressão para os valores de P4 por meio do programa BioEstat (Ayres et al., 2000).

\section{RESULTADOS E DISCUSSÃO}

O número e o percentual de cabras gestantes ou não encontram-se na Tab. 1. O hCG não influenciou o percentual de gestação dos animais $(\mathrm{P}>0,05)$.

A partir dos resultados da Tab. 1, os animais foram agrupados, independentes de raça, em ambos os tratamentos, quando as taxas de gestação foram de 77,2\% e 75,0\% para cabrascontrole e tratadas, respectivamente, não ocorrendo diferença entre tratamentos pelo teste qui-quadrado $(\mathrm{P}>0,05)$. O percentual de animais gestantes foi próximo aos valores obtidos por Fonseca (2002), que verificou, em cabras da raça Alpina tratadas com hCG no quinto dia pósestro, taxas de $84,2 \%$ e $66,7 \%$ nos animais tratados e controle, respectivamente.

A duração da gestação não foi influenciada pelo tratamento, sendo de $151 \pm 2$ dias ( $\mathrm{n}=47$ animais) nas cabras-controle, e $151 \pm 3$ dias $(n=34$ animais) nas tratadas, resultados semelhantes aos encontrados por Robin et al. (1994), de $151,2 \pm 3,2$ dias de gestação.

\footnotetext{
${ }^{1}$ Vetecor ${ }^{\circledR}$, Laboratórios Calier do Brasil Ltda.

${ }^{2}$ Coat-a-count progesterone Kit, DPC, Diagnostic Products

Co. Los Angeles, CA, USA.

${ }^{3}$ Aloka, modelo SSD-500, Tokyo, Japan.
} 
Tabela 1. Percentual e proporção (p) de cabras gestantes de acordo com a raça e o tratamento

\begin{tabular}{|c|c|c|c|c|}
\hline Tratamento & Alpina* (p) & Saanen* (p) & \multicolumn{2}{|c|}{ Total } \\
\hline Controle* & $80,4 \%(37 / 46)$ & $72,7 \%(24 / 33)$ & $77,2 \%$ & $(61 / 79)$ \\
\hline hCG* & $70,3 \%(26 / 37)$ & $81,5 \%(22 / 27)$ & $75,0 \%$ & $(48 / 64)$ \\
\hline Total & $75,9 \%(63 / 83)$ & $74,2 \%(46 / 62)$ & $76,2 \%$ & $(109 / 143)$ \\
\hline
\end{tabular}

* Não houve diferença, pelo teste do qui-quadrado $(\mathrm{P}>0,05)$, entre raças dentro de tratamentos e entre tratamentos.

A taxa de perda fetal nos animais-controle foi de $22,9 \%$ (14 em 61 animais) e de 29,2\% (14 em 48 animais) nos tratados. Valores similares para perdas fetais foram observados nos rebanhos leiteiros da Noruega, isto é, perdas que variaram de 10,0 a 30,0\% (Loken 1990); Melby et al., 1996; Waldeland e Loken, 1991), citados por Engeland et al. (1999). Esses autores mencionaram que a causa de perdas fetais, nãoinfecciosas, em estádios avançados da prenhez, deve-se a distúrbios ocorridos nas funções endócrinas da placenta, pela diminuição da concentração plasmática de estrógeno e pelo aumento da produção do 15-cetodihydroprostaglandina $\mathrm{F}_{2 \alpha}$, metabólito da prostaglandina $\mathrm{F}_{2 \alpha}$.

Quanto à concentração plasmática de $\mathrm{P} 4$ (Tab. 2) não houve diferença entre tratamentos $(\mathrm{P}>0,05)$. Estes resultados são semelhantes aos obtidos por Fredriksson et al. (1984), que verificaram concentrações plasmáticas de $\mathrm{P} 4$ variando de 5 a $10 \mathrm{ng} / \mathrm{ml}$ durante o período de atividade do corpo lúteo, no ciclo estral das cabras (do sétimo dia até a luteólise). As concentrações plasmáticas de P4 apresentaram valores adequados para a manutenção da gestação na espécie caprina.

Tabela 2. Concentração plasmática média de progesterona (P4) em cabras da raça Alpina tratadas com hCG, durante a estação reprodutiva induzida por fotoperíodo artificial

\begin{tabular}{lcc}
\hline Tratamento & Número de amostras & P4 $(\mathrm{ng} / \mathrm{ml})$ \\
\hline Controle & 35 & $5.8388 \mathrm{a}$ \\
hCG & 35 & $5,7679 \mathrm{a}$ \\
\hline
\end{tabular}

Médias seguidas de letras iguais, na coluna, não diferem entre si $(\mathrm{P}>0,05), \mathrm{CV}=55,926$.

Os resultados não confirmam os de Fonseca (2002), que verificou diferenças na concentração plasmática de $\mathrm{P} 4$, em cabras que estavam ciclando naturalmente. Para o autor, os animais tratados com 250UI hCG, mesma dose utilizada nesse experimento, apresentaram maior concentração de P4 em comparação aos animaiscontrole, nos dias 13, 17 e 21 do pós-estro.

Neste estudo, a não-alteração da concentração plasmática de $\mathrm{P} 4$, em função da aplicação do hCG no terceiro dia após o estro, provavelmente ocorreu pela falta de maturidade dos folículos presentes nos ovários e/ou responsividade dos seus receptores. Ginther e Kot (1994) mencionaram que, no dia 0 do ciclo estral, já há folículos em desenvolvimento.

A concentração plasmática de progesterona não diferiu entre os dias 15, 21, 42 e 60 ( $\mathrm{P}>0,05)$, mas houve diferenças entre os dias 0,3 e 8 . A concentração nos dois primeiros dias diferiu da concentração nos demais dias $(\mathrm{P}<0,05)$; a do dia 8 não deferiu da do dia $15(\mathrm{P}>0,05)$ mas diferiu das dos dias 21, 42 e $60 \quad(\mathrm{P}<0,05)$. As concentrações nesses últimos dias não diferiram entre si (Tab. 3; Fig. 1). Este resultado era esperado, pois, com o desenvolvimento e o amadurecimento do corpo lúteo, principalmente por ação do LH, ocorreu aumento na produção de P4 e, conseqüentemente, elevação da concentração plasmática (McCracken et al., 1999), que possibilitou a manutenção da gestação. As concentrações plasmáticas de P4 nos dias 15, 42 e 60 estão dentro da faixa já citada por Frederiksson et al. (1984), assim como as observadas para o dia do estro, mencionadas por Blaszczyk et al. (2003), que verificaram valores de $0,42 \mathrm{ng} / \mathrm{ml}$ durante a estação de acasalamento e de $0,27 \mathrm{ng} / \mathrm{ml}$ fora da estação de acasalamento. A concentração plasmática de P4 do dia 21 foi próxima à encontrada por Sawada et al. (1994) para cabras durante a fase progesterônica do ciclo estral, 7,80ng/ml, demonstrando que o hCG, quando aplicado no terceiro dia após o estro, não é eficaz em aumentá-la. 
Tabela 3. Médias das concentrações plasmáticas de progesterona das cabras da raça Alpina, nos diferentes dias após cobrição, tratadas com hCG, na estação de acasalamento induzida por fotoperíodo artificial

\begin{tabular}{|c|c|c|c|}
\hline \multirow{2}{*}{ Dia } & \multicolumn{3}{|c|}{$\mathrm{P} 4 \mathrm{ng} / \mathrm{ml}$} \\
\hline & Controle (n) & $\mathrm{hCG}(\mathrm{n})$ & Geral (n) \\
\hline 0 & $0,0000 *(5)$ & $0,1603(5)$ & $0,0802 \mathrm{~d}(10)$ \\
\hline 3 & $3,5983(5)$ & $3,4625(5)$ & $3,5304 \mathrm{c}(10)$ \\
\hline 8 & $5,9915(5)$ & $5,5915(5)$ & $5,7915 b(10)$ \\
\hline 15 & $7,6738(5)$ & $7,7042(5)$ & $7,6890 \mathrm{ab}(10)$ \\
\hline 21 & $8,5328(5)$ & $8,6203(5)$ & $8,5766 \mathrm{a}(10)$ \\
\hline 42 & $6,7826(5)$ & $6,8395(5)$ & $6,8110 \mathrm{a}(10)$ \\
\hline 60 & $8,2927(5)$ & $7,9968(5)$ & $8,1447 \mathrm{a}(10)$ \\
\hline $\begin{array}{l}\text { Val } \\
\text { dife } \\
* \text { va }\end{array}$ & dentro da & $\begin{array}{r}\text { seguidos } \\
\text { SNK }(\mathrm{P}<0,\end{array}$ & $\begin{array}{l}\text { letras diferente } \\
\mathrm{CV}=29,034\end{array}$ \\
\hline
\end{tabular}

A melhor curva de ajuste para as concentrações (Y) de P4, dos valores agrupados dos animais controle e tratados, a partir do dia da aplicação da hCG (dia 3), foi a regressão cúbica $\left(\mathrm{Y}=1,48+0,72 * \mathrm{D}-0,023 * \mathrm{X}^{2},+0,00022 * \mathrm{D}^{3}\right.$; $\left.\mathrm{R}^{2}=99,6 \%, \quad \mathrm{P}<0,06\right)$. Os valores da Tab. 3 evidenciam o crescimento da concentração plasmática de $\mathrm{P} 4$ em função dos dias, até o $60^{\circ}$ dia, correspondente ao último dia da coleta de amostras de sangue.

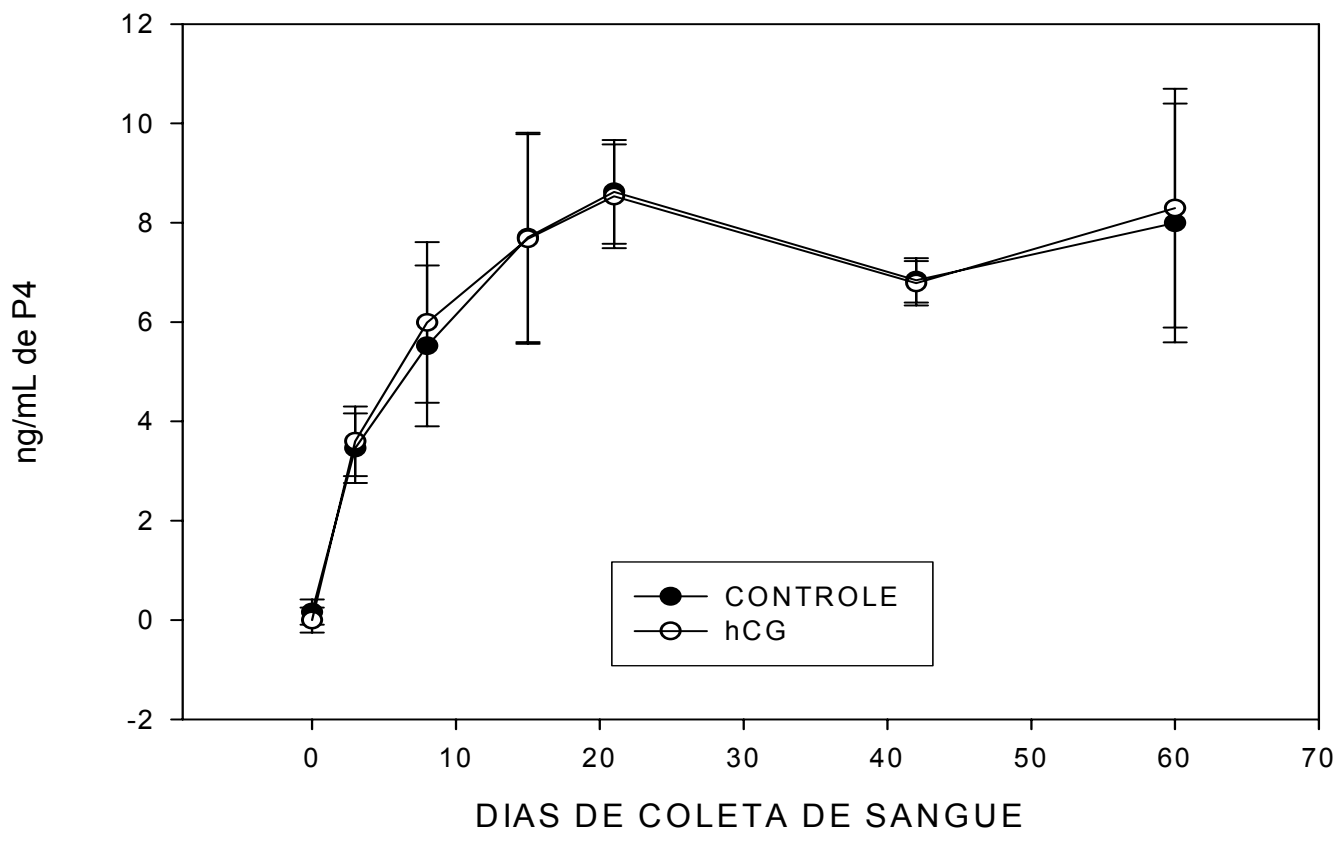

Figura 1. Concentração plasmática de progesterona de cabras da raça Alpina, tratadas com hCG, de acordo com o dia da coleta, durante a estação reprodutiva induzida por fotoperíodo artificial.

\section{CONCLUSÕES}

O hCG aplicado no terceiro dia no pós-estro não alterou a concentração plasmática de $\mathrm{P} 4$, não melhorou a taxa de gestação nem o período de duração da gestação, portanto, não justificou o seu uso em cabras.

\section{REFERÊNCIAS BIBLIOGRÁFICAS}

AYRES, M.; AYRES Jr., M.; AYRES, L.D. et al. BioEstat 2.0. Belém.: Sociedade Civil do Mamirauá-CNPQ, 2000. 258p.

BLASZCZYK, $\quad$ B.; UDALA, J.; GACZARZEWICZ, D. Changes in estradiol, progesterone, melatonin, prolactin and thyroxine 
concentrations in blood plasma of goats following induced estrus in and outside the natural breeding season. Rum. Res., 2003 (in press).

ENGELAND, I.V.; ROPSTAD, E.; KINDAHL, H. et al. Foetal loss in dairy goats: function of the adrenal glands, corpus luteum and the foetalplacental unit. Anim. Reprod. Sci., v.55. p.205$222,1999$.

FONSECA, J.F. Controle e perfil hormonal do ciclo estral e performance reprodutiva de cabras Alpina e Saanen. 2002. 107f. Tese (Doutorado em Zootecnia) - Universidade Federal de Viçosa, Viçosa, MG.

FREDRIKSSON, G.; KINDAHL, H.; EDQVIST, L.E. 11-Ketotetranor PGF metabolites, a suitable indicator for measuring prostaglandin release during the normal oestrus cycle and early pregnancy in the goat. Anim. Reprod. Sci., v.7. p.537-545, 1984.

GINTHER, O.J.; KOT, K. Follicular dynamics during the ovulatory season in goats. Theriogenology, v.42. p.987-1001, 1994.

GORDON, I. Controlled reproduction in sheep $\&$ goats. New York: CAB International, 1997.v.2. 450 p.

HAFEZ, E.S.E. Reprodução animal. 4.ed. São Paulo: Manole, 1988. 720p.

McCRACKEN, J.A.; CUSTER, E.E.; LAMSA, J. Luteolysis: a neuroendocrine-mediated event. Physiol. Rev., v.79, p.263-323, 1999.
MEDAN, M.S.; WATANABE, G.; SASAKI, K. et al. Ovarian dynamics and their association with peripheral concentrations of gonadotropins, ovarian steroids, and Inhibition during the estrous cycle in goats. Biol. Reprod., v.69, p.5763, 2003.

RIBEIRO JÚNIOR, J.I. Análises estatísticas no SAEG. Viçosa: UFV, 2001. 301p.

ROBIN, N.; LAFOREST, J.G.; LUSSIER, J.G. et al. Induction of estrus with intramuscular injections of GnRH or PMSG in lactating goats (Capra hircus) with a progestagen during seasonal anestrus. Theriogenology, v.42, p.107116, 1994.

SAHARREA, A.; VALENCIA, J.; BALCÁZAR, A. et al. Premature luteal regression in goats superovulated with PMSG: effect of hCG or GnRH administration during the early luteal phase. Theriogenology, v.50, p.1039-1052, 1998.

SAWADA, T.; HOU, M.; TAMADA, H. et al. Secretion of progesterone and $20 \alpha$ dihidroprogesterone during the estrous cycle in goats. Steroids, v.59, p.672-675, December 1994.

WALDRON, D.F.; WILLINGHAM, T.D.; THOMPSON, P.V. et al. Effect of concomitant injection of prostaglandin and PMSG on pregnancy rate and prolificacy of artificial inseminated Spanish goats synchronized with controlled internal drug release devices. Small Rum. Res., v.31, p.177-179, 1999. 